\title{
LIDERAZGO ESCOLAR: UNA MIRADA A LOS CRITERIOS DE ÉXITO PEDAGÓGICO DE LOS JEFES DE UNIDAD TÉCNICA PEDAGÓGICA EN CHILE
}

\author{
Felipe Aravena ${ }^{1}$, Marilyn Cádiz ${ }^{2}$, Cinthia Peña ${ }^{3}$, Macarena \\ González $z^{4}$ y Claudio Núñez ${ }^{5}$
}

\section{RESUMEN}

El presente estudio indaga respecto de la importancia del liderazgo ejercido por los jefes de Unidad Técnica Pedagógica (UTP) en relación con los criterios para una clase exitosa. Se desarrolló una metodología de investigación cualitativa que contó con la participación de 27 de estas unidades de las provincias de San Felipe y Los Andes. A partir de una codificación emergente, se desprende que estas jefaturas consideran que una clase exitosa contiene: una estructura claramente definida, un objetivo, la consideración de la diversidad de los estudiantes, la motivación, y el monitoreo y retroalimentación por parte de los docentes. Los resultados de la investigación contribuyen a reflexionar en torno a la generación de programas de liderazgo escolar específicos para los responsables de UTP a lo largo del país.

Conceptos clave: aprendizaje, currículo, liderazgo escolar.

\section{SCHOOL LEADERSHIP: AN APPROACH TO LEARNING SUCCESS CRITERIA OF HEADS OF TECHNICAL-PEDAGOGICAL UNITS (HTPU) IN CHILE}

\section{ABSTRACT}

This study analyzes the importance of the leadership played by the Heads of TechnicalPedagogical Units (UTP, for its Spanish acronym), in terms of the criteria for a successful lesson. A qualitative research study was carried out, with the participation of $27 \mathrm{Heads}$ of UTP from the provinces of San Felipe and Los Andes. From an emergent coding, it is concluded that these academic leaders consider that a successful lesson contains: a clearly identified structure, a learning goal, consideration of student diversity, motivation, and the monitoring process and feedback from teachers. The findings from this research contribute to a reflection on the design of specific professional development programs oriented to UTP.

Key concepts: curriculum, educational leadership, learning.

1 PontificiaUniversidad Católica de Valparaíso, Valparaíso, Chile. Contacto: felipe.aravena@pucv.cl

2 Pontificia Universidad Católica de Valparaíso, Valparaíso, Chile. Contacto: marilyn.cadiz@pucv.cl

3 Pontificia Universidad Católica de Valparaíso, Valparaíso, Chile. Contacto: cinthia.pena@pucv.cl

4 Pontificia Universidad Católica de Valparaíso, Valparaíso, Chile. Contacto: macarena.gonzalez@pucv.cl

5 Investigador independiente, Valparaíso, Chile. Contacto: claudio.labrin95@gmail.com 


\section{Introducción}

Durante la última década en Chile se han observado esfuerzos sostenidos por mejorar la calidad de la educación, poniendo especial énfasis en indicar que el liderazgo escolar es una variable indirecta que afecta el aprendizaje de los estudiantes (Brock \& Grady, 2012; Leithwood, Louis, Anderson \& Wahlstrom, 2004; Marzano, Waters $\&$ McNulty, 2005; Robinson, Hohepa \& Lloyd, 2009). De ahí que el rol de los directores y sus equipos de gestión sea clave en la mejora de los resultados de aprendizaje, así como también en la promoción del desarrollo profesional de los docentes al interior de los establecimientos (Leithwood, Harris \& Hopkins, 2008; Spillane \& Diamond, 2007).

El liderazgo escolar influencia las capacidades, motivaciones y condiciones en que los docentes despliegan sus prácticas pedagógicas en el aula (Hargreaves \& Fink, 2006; Leithwood et al., 2008). Su importancia radica en la posibilidad de modificar los resultados de aprendizaje de los estudiantes de manera indirecta, a través de una influencia directa en las prácticas docentes. De esta forma, los líderes escolares poseen una responsabilidad última en el éxito o fracaso escolar (Spillane $\&$ Lee, 2014). La evidencia empírica internacional y nacional ha reforzado la idea de que es necesario que estos distribuyan el liderazgo (Bolden, 2011; Bush \& Glover, 2012; Harris, 2009; Mansilla y Beltrán, 2016; Quiroga y Aravena, 2017; Torrance, 2013). El liderazgo distribuido abre la posibilidad de pensar en organizaciones más horizontales y democráticas donde el poder, responsabilidades y tareas se comparten, con la finalidad de alcanzar un objetivo común (Camburn, Rowan, \& Taylor, 2003; Harris, 2009). Así, la existencia de más de un líder es necesaria para hacer frente a los múltiples desafíos que presenta el liderar en una escuela (Maureira, Moforte y González, 2014).

Así mismo, la emergencia de nuevos líderes ha reforzado la idea de un liderazgo medio (Bennett, 2006; Harris \& Jones, 2017; Jones \& Harris, 2015). Este es definido por la literatura internacional como el que ejercen los líderes que trabajan al interior del centro escolar directamente con el director, los docentes de aula y los 
estudiantes, es decir, quienes apoyan con su liderazgo pedagógico y formativo a la mejora escolar (Harris \& Jones, 2017). Estos liderazgos poseen un alto impacto directo y positivo en la calidad de la enseñanza y aprendizaje, especialmente, en la construcción de comunidades de aprendizaje profesional (Grootenboer, EdwardsGroves \& Rönnerman, 2015). Al tener contacto directo con docentes y estudiantes, el líder medio tiene más posibilidades de influir de manera directa en la práctica de los docentes en el aula. Por ejemplo, a través de los procesos de acompañamiento, observación de clases, entrega de retroalimentación a las planificaciones e instrumentos de evaluación, entre otros.

En el contexto chileno, el liderazgo escolar ha estado centralizado principalmente en la figura de los directores y de los jefes de Unidad Técnica Pedagógica (UTP) (Carbone, 2008). Este último rol es propio e idiosincrático del contexto chileno, es decir, no se encuentra en otros sistemas internacionales (Flessa, 2014). La presente investigación tiene por objetivo explorar los criterios de éxito pedagógico que los jefes de UTP establecen dentro de sus establecimientos. El estudio considera seis secciones. Las dos primeras presentan evidencia teórica y empírica acerca del rol de los jefes de UTP en el contexto nacional y una revisión teórica en relación con los criterios de éxito pedagógico. La tercera sección da cuenta de la metodología, participantes, procedimientos e instrumentos de recopilación de información. En una cuarta parte se ofrecen los resultados obtenidos en el estudio, para luego discutirlos en la sección siguiente. Finalmente, se presentan las conclusiones, limitantes y proyecciones del estudio a considerar en torno a los criterios de éxito que las jerarquías de UTP poseen en las escuelas chilenas.

\section{El liderazgo de los jefes de UTP: explorando el rol}

En el contexto latinoamericano, Chile es el país que presenta más producción científica respecto de liderazgo escolar (Aravena \& Hallinger, 2018). Sin embargo, la mayoría de las investigaciones se concentra en los directores (entre ellas, Galdames, Montecinos, Campos, Ahumada \& Leiva, 2018; Montecinos, Bush \& Aravena, 2018; Weinstein, Cuellar, Hernández, \& Fernández, 2016; Weinstein 
\& Muñoz, 2014). En consecuencia, la investigación acerca de los jefes de UTP es casi inexistente (Quiroga y Aravena, 2017).

El cargo de jefe de UTP fue creado durante el proceso de municipalización de la educación pública en la década de los ochenta, con el objetivo de fortalecer las funciones directivas dentro de las escuelas y liceos (Núñez, Weinstein y Muñoz, 2010). A este rol se atribuyó la relevancia del liderazgo pedagógico, concentrando las tareas de instrucción (Rodríguez \& Gairín, 2017). Fue concebido como un agente que está directamente involucrado en la creación y desarrollo del currículo, así como de realizar y mantener cambios a largo plazo y resolver situaciones problemáticas que estén relacionadas con los procesos de enseñanza y aprendizaje (Rodríguez \& Gairín, 2017).

En el año 2008, el Ministerio de Educación de Chile, Mineduc, definió el rol de jefe de UTP como "el responsable técnico de la dimensión pedagógica-curricular, liderando procesos de desarrollo de las actividades realizadas en ese ámbito" (Carbone, 2008, p. 17). El fundamento detrás del concepto de "técnico" se relaciona con que estas jefaturas tienen su foco en dos actividades centrales: el seguimiento de los resultados de aprendizaje de los estudiantes y el monitoreo de las prácticas docentes. A su vez, la especificación en lo técnico se caracteriza "porque poseen una disposición positiva a implementar estrategias e instrumentos innovadores para el aprendizaje, aceptan desafíos y están periódicamente actualizando sus saberes y destrezas, mediante la capacitación" (Carbone, 2008, p. 17). En términos de actividades más específicas de su rol, los jefes de UTP se encargan de realizar seguimientos a los procesos curriculares, efectuar un acompañamiento y supervisión al trabajo docente en los procesos de enseñanza-aprendizaje, gestionar recursos tanto humanos como materiales, liderar procesos en donde se implementen metodologías de enseñanza, y asegurar la coherencia de los procesos pedagógicos con el Proyecto Educativo Institucional (PIE) (Carbone, 2008).

Los jefes de UTP son docentes que usualmente llegan a su cargo por invitación directa, o bien, cuando se verifica un cambio 
del equipo directivo completo y el nuevo director trae consigo a este elemento. En un estudio realizado por Contreras (2017) a 850 directivos (350 directores, 250 jefes técnicos y 250 inspectores generales) de establecimientos urbanos, municipales y particulares, se caracterizó a quienes se desempeñan en el rol. Este estudio arrojó que el promedio de edad de docentes ocupando el cargo de UTP es de 48 años. En términos de género, el cargo está marcado mayoritariamente por mujeres con un $68 \%$ del total, mientras que el promedio de años de experiencia laboral es de 23 años, desglosados en ocho en el cargo directivo y 13 años de experiencia en el establecimiento actual (Contreras, 2017).

La caracterización de las prácticas del jefe de UTP establecida por el Mineduc contrasta de forma significativa con la investigación realizada recientemente en el contexto chileno (Cortez y Zoro, 2016; Mansilla y Miranda, 2010). Por su parte, Contreras (2017) concluye que las prácticas más frecuentes desempeñadas por los jefes de UTP tienen relación con supervisar que las clases no sean interrumpidas; revisar evaluaciones de aprendizaje realizadas por docentes; y llevar a cabo entrevistas individuales formales con apoderados. En cambio, las prácticas menos frecuentes corresponden a revisar las planificaciones de clases elaboradas por docentes, resolver problemas relacionados con el reemplazo de profesores y retroalimentar a los docentes respecto de sus prácticas pedagógicas. De forma que las prácticas más frecuentes corresponden a la gestión de la contingencia y las menos, a la gestión del currículo.

Los principales problemas reportados por los jefes de UTP expresan la dificultad de concentrarse en tareas pedagógicas más que administrativas. Dentro de los factores que obstaculizan la gestión pedagógica, se encuentra la falta de liderazgo y la ausencia de prácticas evaluativas centradas en un monitoreo curricular de calidad (Aravena \& Quiroga, 2018; Beltrán, 2014; Cortez y Zoro, 2016; Mansilla y Miranda, 2010). Al respecto, Cortez y Zoro (2016) identifican que una de las complejidades de este rol es poder hacer frente a la excesiva demanda de rendición de cuentas, perdiendo así la oportunidad de contar con mayor tiempo para realizar un trabajo colaborativo con foco en lo pedagógico, en conjunto con el equipo 
docente de su establecimiento. Otra complejidad se encuentra en la distinción entre mecanismos de apoyo y acompañamiento, versus control y presión. Es decir, distinguir cuándo un docente necesita ser apoyado en su práctica y cuándo necesita ser presionado para obtener mejores resultados de aprendizaje. A lo anterior se suma la dificultad de compartir una visión de aprendizaje con el director, lo que dificulta el trabajo colaborativo, la distribución de tareas y el compartir objetivos generales y específicos acerca del futuro de la escuela (Aravena \& Quiroga, 2018).

Por otra parte, para ejercer el cargo de UTP no existe un marco o documento que especifique la formación profesional que estas personas debieran poseer. En contrapartida, Mineduc sí establece directrices específicas respecto del perfil y competencias esperadas en los equipos directivos, considerando al jefe de UTP dentro de ellos. Por ejemplo, esto puede ser observado en referentes como el Marco para la buena dirección y el liderazgo escolar y los Estándares Indicativos de desempeño (Mineduc, 2015). Ambos instrumentos son orientaciones concretas que guían la gestión pedagógica y curricular de los equipos directivos. Sin embargo, no se explicita en ellos qué es una competencia o práctica distintiva del rol de los jefes de UTP.

\section{Criterios de éxito pedagógico}

Los criterios de éxito permiten saber cuándo se ha alcanzado una meta de aprendizaje y, al mismo tiempo, son indicadores que potencian el sentido de autoevaluación, identificando cómo se avanza en relación con las intenciones de aprendizaje (Hattie, 2013). Las intenciones de aprendizaje y los criterios de éxito son fundamentales para determinar el desempeño en una tarea o actividad. Sin los criterios de éxito, es difícil medir el nivel de efectividad alcanzado. Cuando no se reconoce que un éxito es considerado como tal, es más complejo poder determinar los caminos de acción y mejora (Hattie, 2009).

Los criterios de éxito son clave para las escuelas, equipos directivos, docentes y estudiantes. Desde una perspectiva más organizacional, Dweck (2006) señala que las escuelas lograrán el éxito cuando sus estudiantes participen en democracias cada vez 
más diversas y complejas, se envuelvan plenamente en un ambiente dinámico, comuniquen sus ideas a través de una variedad de medios y audiencias, trabajen juntos con otros para resolver problemas complejos, piensen creativamente y lideren su propio aprendizaje y desarrollen disposiciones o modos de pensar, que los empoderen para enfrentar y plantear nuevos desafíos, tomar iniciativas y perseverar a pesar de las dificultades.

Según Martínez, Mcgrath y Foster (2016) las escuelas alcanzan el éxito cuando buscan desarrollar en sus estudiantes las competencias para resolver problemas y situaciones reales usando lo que han aprendido, esto es, transferir lo aprendido en un mundo real, cambiante y altamente complejo. Fullan y Langworthy (2014) proponen que los criterios de éxito en las escuelas siempre debiesen estar expresados en los logros de aprendizaje de los estudiantes, de modo que el éxito pedagógico pueda ser evidenciado a través de lo que han denominado las "6C": creatividad, pensamiento crítico, comunicación, colaboración, carácter y ciudadanía. Ahora bien, desde una perspectiva del aprendizaje, Hattie (2009) identifica cinco componentes que se relacionan con los criterios de éxito en los estudiantes: nivel de desafío, compromiso, confianza, altas expectativas y comprensión conceptual. No obstante, el mayor criterio de éxito para la escuela, los profesores y los líderes, es lograr que los estudiantes participen y disfruten del desafío de aprender que los mantendrá comprometidos con su propio aprendizaje (Fullan \& Langworthy, 2014; Hattie, 2013).

Los criterios de éxito también son determinados por la política pública. En el escenario nacional, destacan dos referentes que cuentan con claros criterios de éxito pedagógico: el Sistema Nacional de Evaluación de Desempeño Profesional Docente (Mineduc, 2017) y el Marco para la buena enseñanza (Mineduc, 2008). El primero contempla una prueba de conocimientos, entrevista por un evaluador par, informe de referencia de terceros, autoevaluación y portafolio, el cual cuenta con criterios de éxito como la formulación de objetivos de clases y su relación con las actividades realizadas. En el apartado de evaluación se considera la correcta elaboración de instrucciones, preguntas, criterios de evaluación y entrega de retroalimentación a 
los estudiantes. Además, en el aspecto de la reflexión se atienden elementos tales como el análisis a partir de las características de los estudiantes y el uso del error como insumo para el aprendizaje. Por otro lado, elementos como la retroalimentación y promoción de la participación a estudiantes, calidad del ambiente de trabajo, calidad del inicio-cierre, y monitoreo de actividades, se expresan como criterios de éxito en la implementación de clases (filmación de una sesión de aprendizaje). Finalmente, en la sección de trabajo colaborativo se debe reflejar el diálogo profesional, ejemplificando ideas que se discutieron, puntos de vista y decisiones que se fueron tomando en el proceso. En este relato, se solicita mostrar aciertos y dificultades dentro del contexto del trabajo colaborativo utilizando ejemplos y detalles que aportan al desarrollo de la idea central.

Por su parte, el Marco para la buena enseñanza establece cuatro dominios que representan aspectos distintivos de la competencia profesional docente. Cada uno de ellos hace referencia a un aspecto distintivo de la enseñanza, siguiendo el ciclo total del proceso pedagógico, desde la planificación y preparación de la clase, la creación de ambientes propicios para el aprendizaje y la enseñanza propiamente tal, hasta la evaluación y la reflexión acerca de la propia práctica docente, necesaria para retroalimentar y enriquecer el proceso. De cada uno de los dominios mencionados se desprenden cinco criterios que indican las prácticas del ejercicio docente, es decir, este marco define un total de 20 prácticas docentes esperadas en un buen proceso de aprendizaje, las que pueden ser entendidas como criterios de éxito (Mineduc, 2008).

\section{Metodología}

El presente estudio es de carácter cualitativo (Chorba, 2011). De esta forma, en una muestra por conveniencia, se encuestó a jefes de UTP de un mismo territorio $(n=27)$, todos ellos participantes de un diplomado implementado por un centro de liderazgo educativo nacional. A estos participantes se les aplicó una encuesta compuesta por cuatro preguntas abiertas y una sección de datos demográficos al iniciar el curso. El objetivo de la encuesta fue recoger percepciones, concepciones e ideas personales acerca de los criterios 
de éxito pedagógico (Stake, 2010). Para luego, desde un enfoque interpretativo, analizar las respuestas utilizando una codificación emergente (Chorba, 2011).

La encuesta constaba con cuatro preguntas abiertas extraídas de Hattie (2013):

- ¿Qué debiese tener una clase exitosa?

- ¿Cómo es un estudiante exitoso?

- ¿Qué hace un buen profesor en una clase?

- ¿Qué elementos contiene una buena tarea de aprendizaje?

Para los fines de este estudio en particular se presentarán solo los resultados de la primera pregunta de la encuesta.

Como resultado de una codificación emergente se levantaron 16 categorías de análisis (Chorba, 2011; Stake, 2010).

\subsection{Participantes}

La muestra estuvo compuesta por 27 participantes, 23 mujeres y cuatro hombres. En términos de edad, el grupo mayoritario se concentra en el rango de 31 y 40 años (44,4\%), seguido de los de más de 51 años $(33,3 \%)$, de entre 41 y 50 años $(14,8 \%)$ y, finalmente, de entre 21 y 30 años $(7,4 \%)$. Los jefes de UTP tienen en promedio 19 años de trayectoria como docentes, en donde el número menor de experiencia es cinco años y el mayor es de 31 años, mientras que los años de experiencia en su rol varían desde los 10 meses hasta los 11 años, con un promedio de cuatro años de experiencia en su cargo. Los jefes de UTP desempeñan su rol en establecimientos de carácter municipal de diferentes contextos, así como de sectores urbanos y rurales.

\subsection{Análisis de datos}

Las preguntas abiertas son relevantes porque permiten explorar un amplio rango de posibles respuestas (Stake, 2010). Siguiendo la propuesta de Erickson, Shaw y Agabe (2007), se analizó cada una de las respuestas de los participantes de forma cualitativa. Cada respuesta 
—entendida como una descripción narrativa— fue desglosada para luego ser considerada como unidad singular de análisis. Como consecuencia lógica, existen más unidades narrativas $(n=94)$ que respuestas de participantes $(n=27)$. En promedio, cada una de las respuestas contuvo 3,4 unidades narrativas. A continuación se presenta un ejemplo:

Pregunta: ¿Qué debiese tener una clase exitosa?

Respuesta: En primer lugar un docente comprometido con su quehacer, con claridad de sus competencias; priorizando ante nada una buena normalización. Lograr los tres momentos (inicio, desarrollo y cierre), y lo más importante fomentar en todo momento el aprendizaje real de sus estudiantes.

Esta descripción narrativa de un encuestado se desglosó en cinco unidades narrativas de la siguiente manera:

a. un docente comprometido con su quehacer.

b. claridad de las competencias docentes.

c. una buena normalización.

d. tres momentos de la clase (inicio, desarrollo y cierre).

e. fomento del aprendizaje real de los estudiantes.

El ejemplo demuestra que la respuesta abierta combina distintos elementos que pueden ser separados en unidades narrativas que se explican en sí mismas y no necesariamente son dependientes unas de otras (Erickson et al., 2007). El proceso de desglosar cada una de las respuestas en unidades narrativas — como propone Chorba (2011) — fue desarrollado de manera independiente por dos investigadores, para luego comparar cada una de las codificaciones, llegando a una convergencia. Las 94 unidades narrativas fueron agrupadas en 16 categorías de análisis. A continuación se presentan las categorías con ejemplos que permiten comprender las respuestas de los participantes. 
Tabla 1

Categorías de análisis y ejemplos de unidades narrativas

\begin{tabular}{|c|c|}
\hline Categoría & Ejemplo de una unidad narrativa \\
\hline 1. Estructura de la clase. & $\begin{array}{l}\text { Una clase exitosa debiese tener siempre una activación } \\
\text { de conocimientos previos, respetar los tiempos de cada } \\
\text { momento (inicio, desarrollo, cierre). }\end{array}$ \\
\hline 2. Objetivo de la clase. & $\begin{array}{l}\text { En donde el objetivo de aprendizaje esté bien planteado y } \\
\text { presente en el transcurso de la clase. }\end{array}$ \\
\hline $\begin{array}{l}\text { 3. Considerar la } \\
\text { diversidad de los } \\
\text { estudiantes. }\end{array}$ & $\begin{array}{l}\text { Es importante que los aprendizajes se desarrollen a partir de } \\
\text { las características de los aprendices y saber cómo aprenden } \\
\text { para así poder organizar la clase. }\end{array}$ \\
\hline 4. Motivación. & $\begin{array}{l}\text { La motivación debe generar impacto en los estudiantes y } \\
\text { capturar su atención. }\end{array}$ \\
\hline $\begin{array}{l}\text { 5. Monitoreo y } \\
\text { retroalimentación. }\end{array}$ & $\begin{array}{l}\text { Un monitoreo constante del docente, siendo un facilitador } \\
\text { con una adecuada retroalimentación. }\end{array}$ \\
\hline 6. Clima de aprendizaje. & $\begin{array}{l}\text { Disciplina. Crear ambientes propicios para que haya } \\
\text { aprendizaje. }\end{array}$ \\
\hline 7. Participación. & $\begin{array}{l}\text { Para ello debe haber una constante participación de los } \\
\text { estudiantes. }\end{array}$ \\
\hline 8. en el aprendizaje. & $\begin{array}{l}\text { Una clase exitosa debiese evidenciar y lograr el aprendizaje } \\
\text { de todos los estudiantes. }\end{array}$ \\
\hline $\begin{array}{l}\text { 9. Actividades } \\
\text { desafiantes. }\end{array}$ & $\begin{array}{l}\text { Actividades desafiantes que logren atender a todos los } \\
\text { estudiantes para lograr la meta. }\end{array}$ \\
\hline $\begin{array}{l}\text { 10. Compromiso } \\
\text { docente. }\end{array}$ & $\begin{array}{l}\text { Un docente comprometido con su quehacer, con claridad } \\
\text { de sus competencias. }\end{array}$ \\
\hline 11. Recursos de aula. & $\begin{array}{l}\text { Los recursos que serán necesarios para que la clase sea } \\
\text { exitosa. }\end{array}$ \\
\hline 12. Claridad en la tarea. & Las actividades deben ser claras. \\
\hline $\begin{array}{l}\text { 13. Considerar el } \\
\text { entorno cultural de los } \\
\text { estudiantes. }\end{array}$ & Identificarse con el entorno cultural o contexto local. \\
\hline $\begin{array}{l}\text { 14. Modelamiento } \\
\text { docente. }\end{array}$ & $\begin{array}{l}\text { El docente debe modelar el trabajo que quiere que realicen } \\
\text { sus alumnos. }\end{array}$ \\
\hline 15. Trabajo colaborativo. & Trabajo grupal y coevaluación. \\
\hline $\begin{array}{l}\text { 16. Planificación } \\
\text { socializada. }\end{array}$ & $\begin{array}{l}\text { Una clase exitosa, debe tener una planificación socializada } \\
\text { tanto por UTP como por el equipo PIE. }\end{array}$ \\
\hline
\end{tabular}

Fuente: Elaboración propia.

\section{Resultados}

A partir de la codificación de respuestas, se observa que la estructura de la clase (17,02\%), el objetivo de la misma $(13,83 \%)$, la consideración de la diversidad de los estudiantes (12,76\%), la motivación (11,70\%), y el monitoreo y retroalimentación $(10,63 \%)$ concentran el mayor número de respuestas, con cerca del 50\% del 
total. En tanto, la claridad en la tarea, considerar el entorno cultural de los estudiantes, el modelamiento docente, el trabajo colaborativo y una planificación socializada concentran el 1,6\%, respectivamente. A continuación se presentan los resultados obtenidos a partir de la codificación emergente.

Tabla 2

Resultados de la codificación de respuestas $(n=27)$

\begin{tabular}{lcc}
\hline \multicolumn{1}{c}{ Categoría de análisis } & $N$ & $\%$ \\
\hline 1. Estructura de la clase & 16 & 17,02 \\
\hline 2. Objetivo de la clase & 13 & 13,82 \\
\hline 3. Considerar la diversidad de los estudiantes & 12 & 12,76 \\
\hline 4. Motivación & 11 & 11,70 \\
\hline 5. Monitoreo y retroalimentación & 10 & 10,63 \\
\hline 6. Clima de aprendizaje & 6 & 6,38 \\
\hline 7. Participación & 6 & 6,38 \\
\hline 8. Foco en el aprendizaje & 5 & 5,31 \\
\hline 9. Actividades desafiantes & 4 & 4,25 \\
\hline 10. Compromiso docente & 3 & 3,19 \\
\hline 11. Recursos de aula & 3 & 3,19 \\
\hline 12. Claridad en la tarea & 1 & 1,06 \\
\hline 13. Considerar el entorno cultural de los estudiantes & 1 & 1,06 \\
\hline 14. Modelamiento docente & 1 & 1,06 \\
\hline 15. Trabajo colaborativo & 1 & 1,06 \\
\hline 16. Planificación socializada & 1 & 1,06 \\
\hline Total & 94 & 100 \\
\hline
\end{tabular}

Fuente: Elaboración propia.

\section{Discusión}

A partir de los datos obtenidos, podemos destacar la importancia que entregan los jefes de UTP a la estructura de la clase para que una esta sea exitosa, atendiendo a los tres momentos (inicio, desarrollo y cierre). Ejemplo de ello son las siguientes unidades narrativas:

Bien definidos los momentos de la clase (inicio, desarrollo, cierre). Lograr los tres momentos (inicio, desarrollo y cierre).

Debe estar planificada, estructurada con sus tres momentos.

Una clase exitosa debiera tener bien claro los tres momentos como inicio, desarrollo y final. 
264 LIDERAZGO ESCOLAR: UNA MIRADA A LOS CRITERIOS DE ÉXITO PEDAGÓGICO DE LOS JEFES DE UNIDAD TÉCNICA PEDAGÓGICA EN CHILE - F. Aravena, M. Cádiz, C.Peña, M. González y C. Núñez

Momentos definidos.

Planificación con una estructura bien clara.

Ahora bien, desde las orientaciones de planificación propuestas por el Mineduc, un docente competente organiza su clase comenzando con un inicio, en donde se espera que se activen los conocimientos previos de los estudiantes, utilizando estrategias que favorezcan la relación con lo que se aprenderá, además de plantear una meta u objetivo que permita motivar o dar sentido al aprendizaje. En el desarrollo, se busca que los docentes realicen actividades de aprendizaje que aborden e instalen el objetivo planteado al inicio y, finalmente, el cierre es el momento donde se sistematiza, evalúa y profundiza el aprendizaje. Este es fundamental para poder recolectar información acerca del nivel de aprendizaje obtenido durante el transcurso de la clase (Mineduc, 2017). Para los jefes de UTP la estructura de una clase contenida en tres momentos es fundamental. No obstante, sabemos que una clase podría cumplir con la estructura, pero no necesariamente generar aprendizajes en sus estudiantes, ya que la mejora escolar no se juega en la estructura, sino en lo que se hace dentro de la sala de clases, aun cuando determinadas estructuras puedan ser obstaculizadores o facilitadores de los procesos de mejora (Bolívar, 2005).

El que la estructura de clase sea el criterio de éxito más frecuente para los jefes de UTP podría explicarse por la influencia de la política pública en el quehacer docente. Específicamente, en los criterios establecidos por la evaluación docente que, hasta la modificación del Sistema Nacional de Evaluación de Desempeño Profesional Docente, solicitaba en su portafolio una planificación de sesiones desarrollada en función de tres momentos. Así también es que en las plataformas ministeriales como Educarchile o Docente Más existen orientaciones y ejemplos de planificación que determinan la estructura de las sesiones en tres momentos.

El segundo criterio de éxito con mayor cantidad de unidades narrativas corresponde al objetivo de la clase $(n=13)$. Según Bolívar (2005) la escuela, en sus esfuerzos por mejorar, adopta políticas centralistas que provienen de estrategias de arriba-abajo. En este caso, 
la política pública promueve que la estructura y un objetivo de clase sean fundamentales para el éxito de la sesión. Sin embargo, estos criterios pueden ser entendidos como residuos de la primera oleada de mejora (Bolívar, 2005), donde se hace difícil distinguir entre el fin y el medio. En este caso, el fin no es la estructura de la clase ni el objetivo de esta, sino que son el medio a través del cual se busca que los estudiantes aprendan. Así, entonces, es posible visualizar un foco centrado en la planificación de momentos y objetivos, pero que no necesariamente incluye oportunidades de aprendizaje para estudiantes. Esto porque se enuncia escasamente cómo esta planificación da cuenta de un foco en el aprendizaje de estudiantes (Fullan \& Langworthy, 2014). Así lo expresan los jefes de UTP:

Una clase exitosa debe, primero que nada estar planificada, con objetivos claros, concretos, alcanzables y desafiantes, además de actividades coherentes que apunten al cumplimiento del objetivo planteado.

Debiese tener una organización temporal de los momentos de la clase definidos y con un objetivo claro y sus actividades coherentes con el objetivo.

Una clase exitosa debe ser coherente entre el OA y las actividades propuestas. Estas últimas deben promover el desarrollo de las habilidades que permitan internalizar el OA.

Cabe destacar que, en esta categoría, se presenta la necesidad de una coherencia entre el objetivo propuesto y las actividades de la sesión (actividades que promuevan aprendizajes). Se puede establecer que si bien se declara y puede existir coherencia entre el objetivo y las actividades propuestas, no necesariamente se desarrollarán aprendizajes profundos por parte de los estudiantes. Por lo que el indicador de coherencia no tiene una relación sine qua non con el aprendizaje de los estudiantes: se requiere considerar la complejidad cognitiva, la comprensión y apropiación de parte de todos los estudiantes y los múltiples factores que inciden en la promoción de aprendizajes de calidad en las aulas y que en las unidades narrativas no se enuncian.

Por otra parte, cuando los jefes de UTP declaran la relevancia de que existan objetivos en la clase para que esta sea exitosa, no se 
extrae de sus discursos si se trata de objetivos acotados o de mayor alcance a una sesión. Los objetivos de aprendizaje declarados en el currículo nacional son comprendidos como ejes que guían el diseño de clases para los docentes. Ellos se definen para cada asignatura, constituyéndose como aprendizajes esperables para cada unidad dentro del año escolar. Se levanta la inquietud, entonces, de cómo estos son gestionados temporalmente y distribuidos en una clase o en un conjunto de ellas, con el fin de lograr los aprendizajes esperados en el transcurso del año, tal como declaran las bases curriculares ministeriales. Cabe preguntarse, pues, cuál es la concepción de aprendizaje que poseen estos jefes de UTP y cómo la comprenden junto con sus docentes, con el fin de planificar y diseñar secuencias de enseñanza y aprendizaje para sus estudiantes. Al respecto, solo cinco jefes de UTP consideran como criterio de éxito el logro de aprendizajes en una clase, es decir, a diferencia de los indicadores de estructura y objetivo de la clase — que suman más del 30\% de las unidades narrativas-, este solo representa un 5\% de las unidades narrativas de los participantes. Se presentan las siguientes unidades como ejemplos:

Y lo más importante: fomentar en todo momento el aprendizaje real de sus estudiantes.

Es importante que los aprendizajes se desarrollen a partir de las características de los aprendices y saber cómo aprenden para así poder organizar la clase.

Desarrollo de habilidades, aprendizaje de contenidos, un aprendizaje profundo.

Dichas actividades deben ser claras y que todo alumno pueda desarrollar.

Una metodología con actividades desafiantes y que logren atender a todos los estudiantes para lograr la meta.

Solo estas acotadas unidades narrativas destacan como elementos de éxito la calidad de las actividades propuestas y el desarrollo de habilidades para los estudiantes. Si bien existe un discurso que otorga importancia a la promoción de aprendizajes, no solo es generalizado, sino además, su identificación como factor de éxito es acotada. 
Como tercera categoría de análisis surge la diversidad de los estudiantes. Esta categoría refiere 12 unidades narrativas, e involucra la multiplicidad de características cognitivas, sociales, emocionales y de intereses que poseen estudiantes. Así es como figuran las siguientes unidades narrativas:

Actividades variadas, utilizando los estilos de aprendizaje.

Una clase exitosa debiera tener actividades pensadas en los niveles visual, auditivo y kinestésico, dando respuesta a la diversidad del grupo curso.

Se incluye la diversidad de estudiantes que esté presente en su sala de clase.

Atención a la diversidad. Actividades adecuadas para las necesidades educativas especiales.

Apuntar a los diferentes ritmos y estilos de aprendizaje.

Clase diversificada en relación con los estudiantes.

El docente debe contemplar actividades para todos los estudiantes que apunten a las diferencias individuales e intereses de los estudiantes; logrando su motivación.

Para los jefes de UTP una clase exitosa requiere, entonces, considerar la diversidad de estudiantes. La política pública contempla este aspecto como un imperativo, puesto "los establecimientos educativos deben asegurar el derecho a la educación de todos y todas las personas de su comunidad, trabajando sobre dos puntos centrales: la eliminación de la discriminación y el abordaje de la diversidad" (Ley No 20.845, 2015). Así mismo, la inclusión a través de prácticas educativas hoy está presente en la identidad de docentes y jefes de UTP al constituir una realidad de los estudiantes del sistema escolar. No obstante, existe una falta de profundización en cuanto a cómo lograr los aprendizajes en relación con este criterio, ya que a pesar de existir nociones respecto de la atención de la diversidad en un aula diversa, no se evidencia cómo lograrlo para promover aprendizajes en los estudiantes que viven en estos contextos. Por el contrario, los criterios están más puestos en lo declarativo que en el cómo incluir a los estudiantes en términos de aprendizaje. 
Cabrera (2007) plantea que "al interior de las escuelas se reconoce que cada niño que asiste a ella tiene una forma diferente o particular de aprender, de afrontar una tarea, es decir, se reconoce que todos tienen ritmos y estilos de aprendizaje diferentes" (p. 1). Este elemento tensiona la atención a la diversidad y la promoción de aprendizajes que conciben los jefes de UTP, ya que en el nuevo escenario educativo donde las diferencias en el procesamiento de información, percepciones y emociones que permiten aprender a los estudiantes, obligan a los docentes a contemplar diseños diversos dependiendo de la contextualización de cada aula, y es allí donde la inclusión es clave. Al parecer, existe consenso en valorar la diversidad — principalmente en términos de estilos de aprendizaje—y concebir las diferencias individuales para aprender, aunque no existe evidencia de que esta diversidad se traduzca en oportunidades para aprender, entendidas estas últimas como una diversificación de estrategias de enseñanza y aprendizaje brindadas en el sistema escolar (Cabrera, 2007).

Ejemplo de esto último es la alusión a las necesidades educativas especiales, las cuales se declaran sin especificar qué recursos se requieren para su atención, cuáles son las necesidades específicas que se abordan, o cómo o por medio de qué estrategias ello se debe llevar a cabo. Este elemento hace referencia a procesos pedagógicos más complejos como las adecuaciones curriculares y la evaluación diferenciada establecidas en el Decreto No 170 (2010) y Decreto No 83 (2017) del Mineduc. Nuevamente emerge aquí la influencia de la política pública de arriba hacia-abajo, que hace fijar normas para determinar quiénes serán los estudiantes con necesidades educativas especiales que se verán beneficiados de las subvenciones para educación especial y las orientaciones técnicas referidas a las estrategias de diversificación de la enseñanza para educación básica, respectivamente. Todo ello permite apoyar su puesta en práctica y la implementación de una enseñanza diversificada en el sistema escolar, pero no necesariamente representa una garantía de calidad de la enseñanza, o bien, de los aprendizajes.

La cuarta categoría de análisis hace referencia a la motivación $(11,7 \%)$, concepto que los jefes de UTP comprenden como un 
agente que logra capturar la atención e interés del estudiante. Se logra desprender que para los participantes del estudio la motivación representa un canal de entrada para iniciar el aprendizaje:

La motivación debe generar impacto en los estudiantes y capturar su atención, para que luego el desarrollo de esta sea efectivo y los estudiantes adquieran los aprendizajes esperados.

Motivación: Que despierte el interés y curiosidad del estudiante. Motivación y altas expectativas del docente hacia sus alumnos.

Tal como se desprende de sus discursos, los jefes de UTP consideran la motivación como un elemento relevante para una clase. Sin embargo, no especifican cómo se espera lograr o cómo esta repercute en el aprendizaje de los estudiantes. El componente motivacional es fundamental para poder alcanzar aprendizajes (Fullan \& Langworthy, 2014; Hattie, 2009). Así mismo, los jefes de UTP requieren tener claridad de que la motivación se asocia con la calidad del diseño de tareas y es parte del proceso, más que un resultado (Hattie, 2013).

La quinta categoría responde al monitoreo y retroalimentación $(10,63 \%)$. En este punto las respuestas discursivas son variadas, ya que un conjunto de unidades narrativas lo relaciona con el proceso del cierre de la clase, como instancia para evidenciar el aprendizaje de estudiantes:

El cierre también debe dar la oportunidad de retroalimentar los o el objetivo de la clase a través de la metacognición.

Interacción pedagógica constante para monitorear el avance de los alumnos, sobre todo la motivación en ellos; plantear preguntas de metacognición al cierre.

Un monitoreo constante del docente, siendo un facilitador. Un cierre adecuado y retroalimentación.

Pese a lo anterior, hay otros participantes que indican que el monitoreo y retroalimentación representa un proceso constante y formativo, atribuyéndole importancia a la retroalimentación y a la evaluación formativa para el aprendizaje, como un proceso que 
permite evidenciar cuánto aprenden los estudiantes y, con ello, ajustar acciones y estrategias que posibiliten aprender más y mejor.

Debe haber una constante participación de los estudiantes, para poder realizar un buen monitoreo de los avances y dificultades y realizar las intervenciones convenientes.

Que se pueda evidenciar que el estudiante aprendió.

Feedback constante.

Debe existir una evaluación formativa permanente.

Donde se realice evaluación formativa constante y exista una autoevaluación.

A partir de las unidades narrativas de esta categoría se establecen dos análisis relevantes. El primero de ellos dice relación con la importancia que los jefes de UTP otorgan a la estructura de la clase. El momento del cierre es entendido como etapa final $-\mathrm{y}$ única- para monitorear, sintetizar, retroalimentar y evidenciar el aprendizaje de estudiantes. Un segundo grupo otorga valor a la retroalimentación y a la evaluación formativa, elementos también presentes en la política pública de los últimos años. La Agencia de la Calidad de la Educación (ACE) propone la evaluación formativa como un proceso que es parte del trabajo cotidiano del aula, utilizada para orientar el proceso de enseñanza-aprendizaje y que permite tomar decisiones oportunas que beneficien a los estudiantes (ACE, 2016). De este modo, la evaluación cumple su propósito formativo, en la medida en que facilita el monitoreo y la retroalimentación para el aprendizaje de los estudiantes.

Es importante destacar que dentro de la categoría de retroalimentación y monitoreo, no existen unidades narrativas alusivas a estrategias concretas que las posibiliten, tales como la utilización del error como medio de aprendizaje, por ejemplo, el que resulta aclaratorio para el contexto de aula, pudiendo trabajar desde allí para desarrollar aprendizaje en los estudiantes (Marzano, 1992).

La sexta categoría de análisis responde al clima de aprendizaje $(6,3 \%)$. Si bien la mayoría indica que se debe desarrollar un buen clima para lograrlo, existen dos tendencias de conceptualización de 
esta categoría. En primer término, la asociada a la normalización de los procesos y de una correcta disciplina al interior del aula. Así, pareciera existir una relación causal entre altos niveles de disciplina y altos niveles de aprendizaje. "El clima, el manejo de la sala y la disciplina son aspectos que suelen considerarse cruciales en el aprendizaje" (Gazmuri, Manzi y Paredes, 2015, p. 118). Desde esta perspectiva, el clima de aprendizaje se asociaría en términos concretos para los jefes de UTP con una sala ordenada conductualmente, conforme el docente establece orden y normaliza el ambiente en la clase.

Priorizando ante nada una buena normalización.

Disciplina. Crear ambientes propicios para que haya aprendizaje.

Por otro lado, se puede apreciar una conceptualización del clima de aprendizaje por parte de los jefes de UTP orientada a la creación de ambientes donde se desarrollan condiciones favorables de aprendizaje, tales como una sana convivencia, lo cual fue un elemento relevado por los participantes de la siguiente forma:

También [debe] existir un buen clima de convivencia en el aula para lograr el objetivo de la clase planificado.

Un clima de convivencia en el que se pueda llevar a cabo lo propuesto.

Buen clima de aula.

Un ambiente de clase que propicie el aprendizaje.

Ahora bien, el ambiente para el aprendizaje no se limita a condiciones materiales, sino que involucra acciones, experiencias, actitudes y condiciones socioafectivas que se producen entre los actores del sistema educativo y el aula para que el aprendizaje se posibilite (Duarte, 2003). De esta forma, resulta interesante que esta categoría sea escasamente relevada, así como también que no se mencione que las normas de convivencia puedan ser consensuadas para que adquieran sentido en los estudiantes. Ello, porque se requiere que los actores que participan en el proceso se vinculen a través de un trabajo en equipo que potencie las dinámicas de relación entre los sujetos y permita formas de relación cálidas, amables, asertivas y proactivas (Florez, Castro, Galvis, Acuña y Zia, 2017). 
Asociada a lo anterior, encontramos una séptima categoría (o subcategoría) denominada participación (6,3\%), que refiere a la interacción de los estudiantes y la interacción profesor-alumno, en espacios efectivos que promuevan y posibiliten el aprendizaje, como se ejemplifica en las siguientes unidades:

Una interacción con todos los alumnos.

Dando también la posibilidad de interacción de los alumnos con sus pares y docentes.

Interacción permanente docente-alumno.

Se considera aquí un ambiente socioafectivo construido sobre la base de relaciones sociales donde conviven e interactúan docentes y estudiantes. Para Florez et al. (2017), "constituyen espacios importantes para el desarrollo emocional de los estudiantes, debido a que los procesos de comunicación abren la posibilidad real de interactuar con el otro" (p. 83).

Por su parte, las actividades desafiantes (4,25\%) son entendidas por los jefes de UTP como actividades congruentes con el objetivo de la clase, o bien, que permiten desarrollar habilidades de pensamiento complejo.

Actividades desafiantes y que permitan al estudiante involucrarse en el logro del objetivo.

[Actividades] alcanzables y desafiantes.

En estas afirmaciones se evidencia una escasa conceptualización en torno a qué constituye en sí una actividad desafiante y cómo pueden promover aprendizajes profundos en estudiantes: en efecto, una actividad podría ser desafiante solo en la medida en que se relaciona con un objetivo planteado para la sesión; o bien, en tanto desarrolla habilidades de pensamiento complejo o, por lo menos, declara desarrollar. Cabe destacar que la importancia de este criterio de éxito puede relacionarse con su inclusión en el Marco para la buena enseñanza, documento que los jefes de UTP conocen como guía fundamental de la política pública, el cual en su dominio señala: "C: Enseñanza para el aprendizaje de todos los estudiantes" y 
en su criterio $\mathrm{C} 2$, plantea como descriptor que cada docente "propone actividades que involucran cognitiva y emocionalmente a los estudiantes y entrega tareas que los comprometen en la exploración de los contenidos" (Mineduc, 2008, p. 14).

La décima categoría de análisis hace referencia al compromiso docente (3,19\%). Los resultados evidencian aquí dos elementos que podrían reflejar este compromiso. El primero de ellos tiene relación con el "empoderamiento" que evidencie el docente (respecto de los saberes disciplinarios, pedagógicos, prácticas u otros elementos), en tanto que una segunda característica se relaciona con la motivación con su trabajo o compromiso en su quehacer.

Además de un docente empoderado y motivado en su trabajo.

En primer lugar un docente comprometido con su quehacer, con claridad de sus competencias.

La última categoría de análisis con más de una unidad narrativa, corresponde a los recursos de aula (3,19\%). Los jefes de UTP los comprenden como las condiciones materiales para que se desarrolle una clase efectiva, sin considerar o describir mayormente qué tipo de materiales de apoyo debiesen existir, de qué calidad o con qué finalidad pedagógica debieran presentarse.

Los recursos que serán necesarios para que la clase sea exitosa. Para una clase efectiva debe existir el material de apoyo.

Las últimas categorías de éxito poseen una única referencia, es decir, la inclusión de estos criterios de éxito corresponde a unidades narrativas aisladas de los jefes de UTP, mismas que no logran constituirse ni como indicadores de aprendizaje, ni como elementos que posibiliten el éxito de una sesión. Entre ellas se cuentan: claridad en la tarea, consideración del entorno cultural de los estudiantes, modelamiento docente, trabajo colaborativo y una planificación socializada. 
274 LIDERAZGO ESCOLAR: UNA MIRADA A LOS CRITERIOS DE ÉXITO PEDAGÓGICO DE LOS JEFES DE UNIDAD TÉCNICA PEDAGÓGICA EN CHILE - F. Aravena, M. Cádiz, C.Peña, M. González y C. Núñez

\section{Conclusiones}

Los jefes de UTP están encargados de monitorear y retroalimentar las prácticas docentes con foco en el aprendizaje de los estudiantes. En distintas ocasiones pueden perder ese norte concentrando su energía, tiempo y capacidades en rendir cuentas acerca de las prácticas docentes, más que acompañando a los profesores para que promuevan mejores experiencias, ambientes y resultados de aprendizaje entre sus estudiantes (Aravena \& Quiroga, 2018; Contreras, 2017). Es decir, las labores administrativas y de rendición de cuentas pueden tensionar el liderazgo pedagógico de estas jefaturas.

Los resultados de la investigación señalan claramente que los jefes de UTP consideran como criterios de una clase exitosa: una clase estructurada con tres momentos claramente diferencias (inicio, desarrollo y cierre), con un claro objetivo de aprendizaje, que considera la diversidad de los estudiantes, promueve la motivación, el monitoreo y la retroalimentación. Los criterios de éxito tienen un claro énfasis en lo que hacen los docentes más que lo que aprenden los estudiantes. Así, pareciera ser que el enfoque para los jefes de UTP no es el aprendizaje, sino la enseñanza de los docentes, puesto que tal como hemos visualizado en los criterios establecidos por los participantes del estudio, un 65,93\% de las afirmaciones se concentra en dichos elementos como los principales indicadores de éxito, y solo un 5,3\% de los criterios apuntan explícitamente al aprendizaje. En la medida en que los jefes de UTP cuenten con criterios de éxito pedagógico asociados al aprendizaje de los estudiantes, las posibilidades de influenciar en las prácticas pedagógicas serán mayores (Hattie, 2013).

Pareciera ser que frente a las responsabilidades declaradas por Mineduc en relación con supervisar y acompañar el trabajo de los docentes, asegurar la implementación de metodologías y prácticas pedagógicas en el aula y realizar seguimiento de los procesos curriculares, los jefes de UTP han puesto su atención en la enseñanza de los docentes, más que en el aprendizaje de los estudiantes. 
Desde esta perspectiva, los criterios de éxito sirven para orientar dónde focalizar la mirada, pero si los jefes de UTP siguen manteniendo el énfasis de su quehacer en los docentes, sin considerar los aprendizajes de los estudiantes, difícilmente se podrán movilizar la práctica pedagógica.

Lo anterior evidencia que es necesario contar con programas de formación que vinculen la teoría y la práctica a través de tareas auténticas y contextualizadas con el desempeño del rol de los jefes de UTP, haciendo hincapié en el proceso de aprendizaje (Cortez y Zoro, 2016; Quiroga y Aravena, 2017). Dichos programas requieren, al mismo tiempo, considerar una reflexión profunda respecto de los criterios de éxito en función del aprendizaje, ya que distinguir entre el fin y el medio, es decir, entre los propósitos y las estrategias, resulta clave. En términos concretos, esto significa diferenciar entre las secuencias que componen una clase y el aprendizaje de los estudiantes.

Un líder pedagógico que posea claridad que el éxito escolar se mide en términos de abrir más oportunidades de aprendizaje para sus estudiantes tendrá, a su vez, para sí mismo más oportunidades de impactar en el mejoramiento de las prácticas docentes y, finalmente, influenciar y movilizar a su equipo profesional.

\section{Referencias}

Agencia de la Calidad de la Educación, ACE. (2016). Cuadernillo de orientaciones para la calidad de la educación. Santiago de Chile: Ministerio de Educación de Chile, Mineduc.

Aravena, F. \& Hallinger, P. (2018). Systematic review of research on educational leadership and management in Latin America, 1991-2017. Educational Management Administration \& Leadership, 46(2), 207-225. https://doi. org/10.1177/1741143217745882

Aravena, F. \& Quiroga, M. (2018). Bicephalous leadership structure: an exploratory study in Chile. International Journal of Leadership in Education, 22(6), 670-684. https://doi.org/10.1080/13603124.2018 .1492022

Beltrán, J. (2014). Factores que dificultan la gestión pedagógica curricular de los jefes de Unidades Técnico Pedagógicas. Revista Mexicana de 
276 LIDERAZGO ESCOLAR: UNA MIRADA A LOS CRITERIOS DE ÉXITO PEDAGÓGICO DE LOS JEFES DE UNIDAD TÉCNICA PEDAGÓGICA EN CHILE - F. Aravena, M. Cádiz, C.Peña, M. González y C. Núñez

Investigación educativa, 19(62), 939-961. Recuperado de http://www. redalyc.org/articulo.oa?id=14031461014

Bennett, N. (2006). Making a difference. A study of effective middle leadership in schools facing challenging circumstances. Nottingham: College for School Leadership.

Bolden, R. (2011). Distributed leadership in organizations: A review of theory and research. International Journal of Management Reviews, 13(3), 251269. https://doi.org/10.1111/j.1468-2370.2011.00306.x

Bolívar, A. (2005). ¿Dónde situar los esfuerzos de la mejora? Política educativa, escuela y aula. Educação \& Sociedade, 26(92), 859-888. https://doi. org/10.1590/s0101-73302005000300008

Brock, B. \& Grady, M. (2012). The daily practices of successful principals. California: SAGE.

Bush, T. \& Glover, D. (2012). Distributed leadership in action: Leading highperforming leadership teams in English schools. School Leadership \& Management, 32(1), 21-36. https://doi.org/10.1080/13632434.2011. 642354

Cabrera, E. (2007). Dificultades para aprender o dificultades para enseñar. Revista Iberoamericana de Educación, 43(3), 1-7. https://doi. org/10.35362/rie4332327

Camburn, E., Rowan, B., \& Taylor, J. E. (2003). Distributed leadership in schools: The case of elementary schools adopting comprehensive school reform models. Educational Evaluation and Policy Analysis, 25(4), 347-373. https://doi.org/10.3102/01623737025004347

Carbone, R. (2008). Situación del liderazgo educativo en Chile. Santiago de Chile: Ministerio de Educación de Chile, Mineduc y Universidad Alberto Hurtado.

Chorba, K. (2011). A review of qualitative research: Studying how things work. The Qualitative Report, 16(4), 11361140. Recuperado de http://citeseerx.ist.psu.edu/viewdoc/ download?doi=10.1.1.1031.4453\&rep=rep1\&type=pdf

Contreras, D. (2017). Liderazgo escolar en Chile: una mirada a las prácticas directivas. Evidencias, 34. Santiago de Chile: Centro de Estudios, Mineduc. Recuperado de http://liderazgoescolar.mineduc.cl/wpcontent/uploads/sites/55/2017/02/EVIDENCIAS-34.pdf

Cortez, M. y Zoro, B. (2016). El rol estratégico de los liderazgos medios al interior de las escuelas para la mejora escolar: una mirada desde la literatura internacional. Nota Técnica N 8, Líderes Educativos, Centro de Liderazgo para la Mejora Escolar: Pontificia Universidad Católica de 
Valparaíso, Chile. Recuperado de https://www.lidereseducativos.cl/ wp-content/uploads/2017/01/NT-8.pdf

Decreto Diversificación de la Enseñanza $N^{\circ} 83$ del Ministerio de Educación. Diario Oficial de la República de Chile, 1, junio de 2015. Recuperado desde http://especial.mineduc.cl/wp-content/uploads/ sites/31/2016/08/Decreto-83-2015.pdf

Decreto Normas para Determina los Alumnos con Necesidades Educativas Especiales que Serán Beneficiarios de las Subvenciones para Educación Especial $N^{\circ} 170$ del Ministerio de Educación. Diario Oficial de la República de Chile, 21, abril de 2017. Recuperado desde http://especial. mineduc.cl/wpcontent/uploads/sites/31/2016/08/201304231500550. DEC200900170.pdf

Duarte, J. (2003). Ambientes de aprendizaje. Una aproximación conceptual. Estudios Pedagógicos (Valdivia), 29, 97-113. https://doi.org/10.4067/ s0718-07052003000100007

Dweck, C. (2006). Mindset. New York: Random House.

Erickson, A., Shaw, J., \& Agabe, Z. (2007). An empirical investigation of the antecedents, behaviors, and outcomes of bad leadership. Journal of Leadership Studies, 1(3), 26-43. https://doi.org/10.1002/jls.20023

Flessa, J. (2014). Learning from school leadership in Chile. Canadian and International Education, 43(1). Recuperado de http://ir.lib.uwo.ca/cgi/ viewcontent. cgi? article $=1318 \&$ context $=$ cie-eci

Florez, R. Castro, J., Galvis, D., Acuña, L., y Zia, L. (2017). Ambientes de aprendizaje y sus mediaciones en el contexto educativo de Bogotá. Bogotá: Instituto para la Investigación Educativa y el Desarrollo Pedagógico, IDEP.

Fullan, M. \& Langworthy, M. (2014). A rich seam. How new pedagogies find deep learning. London: Pearson.

Galdames, S., Montecinos, C., Campos, F., Ahumada, L., \& Leiva, M.V. (2018). Novice principals in Chile mobilizing change for the first time: Challenges and opportunities associated with a school's readiness for change. Educational Management Administration E Leadership, 46(2), 318-338. https://doi.org/10.1177/1741143217707520

Gazmuri, C., Manzi, J., y Paredes, R. (2015) Disciplina, clima y desempeño escolar en Chile. Revista CEPAL, 115, 115-128. https://doi. org/10.18356/bc44a854-es 
278 LIDERAZGO ESCOLAR: UNA MIRADA A LOS CRITERIOS DE ÉXITO PEDAGÓGICO DE LOS JEFES DE UNIDAD TÉCNICA PEDAGÓGICA EN CHILE - F. Aravena, M. Cádiz, C.Peña, M. González y C. Núñez

Grootenboer, P., Edwards-Groves, C., \& Rönnerman, K. (2015). Leading practice development: Voices from the middle. Professional Development in Education, 41(3), 508-526. https://doi.org/10.1080/19415257.201 4.924985

Hargreaves, A. \& Fink, D. (2006). Sustainable leadership. San Francisco: Jossey-Bass Education Series.

Harris, A. (2009). Distributed leadership, different perspectives. London: Springer.

Harris, A. \& Jones, M. (2017). Middle leaders matter: Reflections, recognition, and renaissance. School Leadership \& Management, 37(3), 213-216. https://doi.org/10.1080/13632434.2017.1323398

Hattie, J. (2009). Visible learning: A synthesis of 800 meta-analyses on achievement. London: Routledge.

Hattie, J. (2013). Visible learning for teachers: Maximizing impact on learning. London: Routledge.

Jones, M. \& Harris, A. (2015). Professional learning as community. London: Professional Development Today.

Leithwood, K., Louis, K. S., Anderson, S., \& Wahlstrom, K. (2004). How leadership influences student learning. New York: Wallace Foundation.

Leithwood, K., Harris, A., \& Hopkins, D. (2008). Seven strong claims about successful school leadership. School Leadership and Management, 28(1), 27-42. https://doi.org/10.1080/13632430701800060

Ley N 20.845 de Inclusión Escolar que Regula la Admisión de los y las Estudiantes, Elimina el Financiamiento Compartido y Prohíbe el Lucro en Establecimientos Educacionales que Reciben Aportes del Estado. Diario Oficial de la República de Chile, Santiago, Chile, 8 de junio de 2015. Recuperado de https://www.leychile.cl/ Navegar?idNorma $=1078172$

Mansilla, J. y Beltrán, J. (2016). Racionalidad instrumental y prácticas de gestión en jefes técnicos de liceos de la Araucanía. Revista de Investigación Educativa, 34(1), 151-165. https://doi.org/10.6018/ rie.34.1.218041

Mansilla, J. y Miranda, J. (septiembre, 2010). Las unidades técnicas pedagógicas en la enseñanza media: razón administrativa y razón pedagógica en contextos secundarios vulnerables. Trabajo presentado en el Congreso Iberoamericano de Educación, Metas 2021. Buenos Aires, Argentina. 
Martínez, M., McGrath, D., \& Foster, E. (2016). How deeper learning can create a new vision for teaching. Arlington: National Commission on Teaching $\&$ America's Future (NCTAF).

Marzano, R. J. (1992). A different kind of learning: Teaching with dimensions of learning. Alexandria: Association for Supervision and Curriculum Development.

Marzano, R. J., Waters, T., \& McNulty, B.A. (2005). School leadership that works. Alexandria: Association for Supervision and Curriculum Development.

Maureira, O., Moforte, C., y González, G. (2014). Más liderazgo distribuido y menos liderazgo directivo: nuevas perspectivas para caracterizar procesos de influencia en los centros escolares. Perfiles Educativos, 36(146), 134-153. https://doi.org/10.22201/ iisue.24486167e.2014.146.46033

Ministerio de Educación de Chile, Mineduc. (2008). Marco para la buena enseñanza. Recuperado de https://www.docentemas.cl/docs/MBE2008. pdf

Ministerio de Educación de Chile, Mineduc. (2015). Marco para la buena dirección y liderazgo escolar. Recuperado de http://liderazgoescolar. Mineduc.cl/wp-content/uploads/sites/55/2016/04/MBDLE_2015.pdf

Ministerio de Educación de Chile, Mineduc. (2015). Estándares indicativos de desempeño. Recuperado de http://archivos.agenciaeducacion.cl/ documentos-web/Estandares_Indicativos_de_Desempeno.pdf

Ministerio de Educación de Chile, Mineduc. (2017). Docente más. Sistema Nacional de Evaluación de Desempeño Profesional Docente. Recuperado de http://www.docentemas.cl/

Montecinos, C., Bush, T., \& Aravena, F. (2018). Moving the school forward: Problems reported by novice and experienced principals during a succession process in Chile. International Journal of Educational Development, 62, 201-208. https://doi.org/10.1016/j. ijedudev.2018.04.004

Núñez, I., Weinstein, J., y Muñoz, G. (2010). ¿Posición olvidada? Una mirada desde la normativa a la historia de la dirección escolar en chile. Psicoperspectivas, 9(2), 53-81. https://doi.org/10.5027/ psicoperspectivas-vol9-issue2-fulltext-117

Quiroga, M. y Aravena, F. (2017). Jefes de UTP nóveles en Chile: desafíos del liderazgo pedagógico. Revista Perspectiva Educacional, 56(3), 76-97. http://dx.doi.org/10.4151/07189729-Vol.56-Iss.3-Art.506 
Robinson, V., Hohepa, M., \& Lloyd, C. (2009). School leadership and student outcomes: Identifying what works and why: Best evidence synthesis iteration [BES]. Wellington: New Zealand Ministry of Education.

Rodríguez, G. \& Gairín, J. (2017). Influence of the practices of pedagogical leadership in the educational pedagogical practices: Case in Chile of the Pedagogical Technical Units. International Journal of Educational Leadership and Management, 5(1), 6-29. http://dx.doi.org/10.17583/ ijelm.2017.2469

Spillane, J. P. \& Diamond, J. (2007). Distributed leadership in practice. New York: Teachers College Press.

Spillane, J. \& Lee, L. (2014). Novice school principals' sense of ultimate responsibility: Problems of practice in transitioning to the principal's office. Educational Administration Quarterly, 50(3), 431-465. https:// doi.org/10.1177/0013161x13505290

Stake, R. E. (2010). Qualitative research: Studying how things work. New York: The Guilford Press.

Torrance, D. (2013). Distributed leadership: Challenging five generally held assumptions. School Leadership \& Management, 33(4), 354-372. https:// doi.org/10.1080/13632434.2013.813463

Weinstein, J., Cuellar, C., Hernández, M., y Fernández, M. (2016). Director(a) por primera vez. Un estudio sobre la experiencia y socialización de los directores noveles en establecimientos municipales en Chile. Revista Calidad en la Educación, 44, 12-45. https://doi.org/10.4067/s071845652016000100002

Weinstein, J. \& Muñoz, G. (2014). When duties are not enough: Principal leadership and public or private school management in Chile. School Effectiveness and School Improvement, 25(4), 651-670. https://doi.org/ $10.1080 / 09243453.2013 .792850$

Recibido: 24/04/2019

Aceptado: 23/09/2019 